\title{
Aeroelastic Modeling and Control of an Experimental Flexible Wing
}

\author{
Manuel Pusch*, Daniel Ossmann ${ }^{\dagger}$ and Thiemo Kier ${ }^{\ddagger}$ \\ Institute of System Dynamics and Control, German Aerospace Center (DLR), 82234 Weßling, Germany \\ Martin Tang ${ }^{\S}$, Jannis Lübker ${ }^{\text {Il }}$ and Johannes Dillinger" \\ Institute of Aeroelasticity, German Aerospace Center (DLR), 37073 Göttingen, Germany
}

\begin{abstract}
Structural weight reduction and high aspect ratio wings play a key role in improving the performance of modern transport aircraft. This leads to a highly flexible aircraft structure which is sensitive to external disturbances like gusts. To counteract this undesired effect, active control is a promising technology. In this paper, a gust load alleviation controller is designed for a wind tunnel model of a flexible wing with various trailing edge flaps and acceleration sensors. For a sophisticated model-based controller design, a detailed aeroelastic model is derived describing the coupling of structural dynamics and aerodynamics. Additionally, actuator dynamics and structural modes are identified and used to improve model accuracy. Subsequently, the weakly damped first wing bending mode, which causes high structural loads, is isolated via $\mathcal{H}_{2}$-optimal blending of control inputs and measurement outputs. In this way, a gain-scheduled single-input single-output controller can be designed to control the desired aeroelastic mode. Eventually, the great potential of the proposed control approach is verified by a wind tunnel test including different gust excitations and varying airspeeds.
\end{abstract}

\section{Introduction}

In order to improve aircraft performance, structural weight reduction and aerodynamically efficient high aspect ratio wings play a key role [1]. This, however, typically leads to an increased flexibility of the aircraft structure with an increased sensitivity to gust encounters [2]. To counteract this adverse effect, an active gust load alleviation (GLA) control system can be used which feeds back multiple measurements to available control surfaces. The actual effect of gusts on highly flexible flexible wings and the capabilities of different GLA systems can be evaluated at low cost by means of wind tunnel experiments [3, 4]. In [5], an adaptive control strategy for damping the first wing bending mode has been extensively tested in a wind tunnel. Another series of wind tunnel studies characterizing the system response and active control characteristics has been carried out on the so-called "SensorCraft", see [6-8]. As the wind tunnel tests confirm, the performance of a GLA system largely depends on available measurements and control surfaces. Hence, increasing the number of sensors and (multifunctional) control surfaces allows improving GLA performance [9], but also leads to new challenges in the design and certification of the control system [10].

In [11], a new control approach has been presented for aeroelastic systems with a large number of control inputs and measurement outputs. In order to control individual aeroelastic modes, the approach reduces the complexity of the control design problem by blending the inputs and outputs beforehand. The sensor signals are weighted and summed up in a way such that the resulting (virtual) measurement signal represents the response of the mode of interest. Similarly, available control surfaces are allocated such that the considered mode can be controlled explicitly by a single (virtual) control signal. As a result, individual modes can efficiently be isolated and hence controlled by means of a single-input and single-output (SISO) controller.

While in [11], the performance of the proposed control approach has been verified using time and frequency domain simulations, the goal of this paper is to verify these results by means of a wind tunnel experiment. To that end, a model of a flexible wing of $1.6 \mathrm{~m}$ span is designed and augmented with three trailing edge flaps and multiple acceleration

\footnotetext{
*Research Fellow, Department of System Dynamics and Control, manuel.pusch@dlr.de

${ }^{\dagger}$ Research Scientist, Department of System Dynamics and Control, daniel.ossmann@dlr.de, AIAA member

$\doteqdot$ Research Fellow, Department of System Dynamics and Control, thiemo.kier@dlr.de

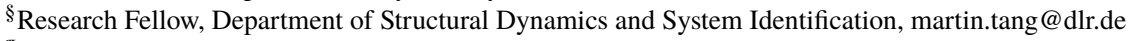

IResearch Fellow, Department of Aeroelastic Experiments, jannis.luebker@dlr.de

" Research Scientist, Department of Loads Analysis and Aeroelastic Design, johannes.dillinger@dlr.de
} 
measurements. For model-based controller design and tuning, an aeroelastic model of the flexible wing is derived in Section II, which captures the coupling of unsteady aerodynamics with structural dynamics. Furthermore, acceleration and load measurements as well as identified actuator dynamics are added to the model. Subsequently, the first wing bending mode is isolated by blending the control inputs and measurement outputs according to [11]. Based on that, a SISO controller, which is scheduled with airspeed, is designed and tuned to achieve a maximum damping of the first wing bending mode during gust encounters. In Section III, the GLA controller design procedure is described and the achieved control performance is evaluated in simulation. Eventually, the effectiveness of the proposed GLA system is verified by wind tunnel experiments as described in Section IV.

\section{Aeroelastic Modeling}

In this section, the modeling procedure for the experimental flexible wing is described. Individual models of the aerodynamics, the structural dynamics and the actuator dynamics are derived and interconnected. The resulting integrated aeroelastic model includes multiple measurement outputs and flap deflection command inputs as well as an input for simulating vertical gust excitations. The general modeling procedure is based on [12] and depicted In Figure 1, where the individual parts are explained in the following subsections.

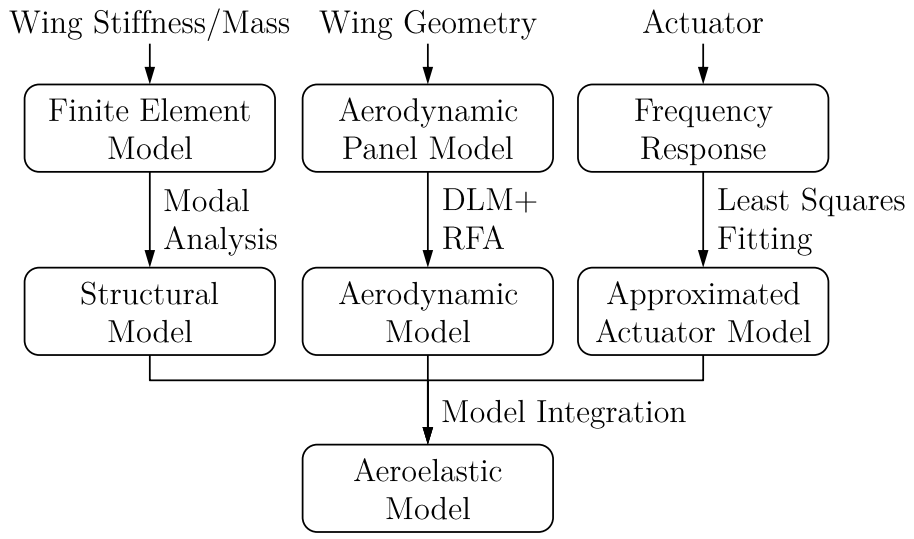

Fig. 1 Aeroelastic modeling procedure.

\section{A. Structural Model}

From the aeroelastic-tailoring process described in [13] and applied in [14], a stiffness-optimized finite element model (FEM) of the flexible wing is obtained. Condensing the FEM and carrying out a modal analysis yields the structural model

$$
G_{\text {struct }}(s)=\frac{1}{M_{h, h} s^{2}+B_{h, h} s+K_{h, h}}
$$

described in the modal or generalized coordinates $h$. In Equation (1), $s$ denotes the Laplace variable and $M_{h, h}, B_{h, h}$ and $K_{h, h}$ describe the modal mass, damping and stiffness matrix, respectively. Applying (generalized) external loads $p_{h}^{(\text {ext })}$ on the wing structure, the resulting modal deflections are given as

$$
x_{h}=G_{\text {struct }}(s) p_{h}^{(\mathrm{ext})}
$$

where $x_{h}$ captures both the displacements of rigid body and the flexible modes. For the considered flexible wing, the rigid body motion is constrained as the wing is clamped in the wing root. Solely the rotation around the quarter chord line of the wing is not fixed, which is used to simulate a gust excitation, see Section II-C for more details. Furthermore, only the ten flexible modes with the lowest natural frequency are considered in the structural model while the remaining higher-frequent modes are truncated. 


\section{B. Aerodynamic Model}

The aerodynamic model $Q_{\text {aero }}(s)$ describes the aerodynamic loads $p^{(\text {aero) }}$ resulting from modal deflections $x_{h}$ and control surface deflections $\delta$. The aerodynamic loads can be partitioned into the generalized loads $p_{h}^{\text {(aero) }}$ acting on the wing structure and the loads $p_{c}^{(\text {aero) }}$ occurring at predefined sensor positions. Based on that, it can be written

$$
p^{\text {(aero) }}=\left[\begin{array}{l}
p_{h}^{\text {(aero) }} \\
p_{c}^{\text {(aero) }}
\end{array}\right]=\left[\begin{array}{ll}
Q_{h, h}(s, V) & Q_{h, \delta}(s, V) \\
Q_{c, h}(s, V) & Q_{c, \delta}(s, V)
\end{array}\right]\left[\begin{array}{c}
x_{h} \\
\delta
\end{array}\right]=Q_{\text {aero }}(s, V)\left[\begin{array}{c}
x_{h} \\
\delta
\end{array}\right],
$$

where $Q_{\text {aero }}(s)$ is partitioned into the the four individual transfer function matrices $Q_{h, h}(s), Q_{h, \delta}(s), Q_{c, h}(s)$ and $Q_{c, \delta}(s)$. In order to capture also unsteady aerodynamics, $Q_{\text {aero }}(s)$ is computed in the frequency domain using the doublet lattice method (DLM) [15]. To that end, the lifting surfaces are discretized by aerodynamic panels, where the panel size of the trailing edge flaps is reduced for a higher modeling accuracy, see Figure 2. In order to obtain an unsteady aerodynamic model which can also be used for time domain simulations, a rational function approximation (RFA) according to Roger [16] is carried out yielding

$$
Q_{\text {aero }}(s, V)=\frac{1}{2} \rho V^{2}\left[D_{\text {aero }}^{(0)}+\left(\frac{s c}{2 V}\right) D_{\text {aero }}^{(1)}+\left(\frac{s c}{2 V}\right)^{2} D_{\text {aero }}^{(2)}+C_{\text {aero }}\left(\left(\frac{s c}{2 V}\right) I-A_{\text {aero }}\right)^{-1} B_{\text {aero }}\right],
$$

where $c$ denotes the reference chord length. Certainly, the unsteady aerodynamic model depends on the velocity of the surrounding air flow $V$ and its density $\rho$. Recalling that $Q_{\text {aero }}(s)$ actually consists of four different parts, the given matrices in Equation (4) can also be partitioned as

$$
B_{\text {aero }}=\left[\begin{array}{ll}
B_{h} & B_{\delta}
\end{array}\right], C_{\text {aero }}=\left[\begin{array}{l}
C_{h} \\
C_{c}
\end{array}\right] \text { and } D_{\text {aero }}^{(d)}=\left[\begin{array}{ll}
D_{h, h}^{(d)} & D_{h, \delta}^{(d)} \\
D_{c, h}^{(d)} & D_{c, \delta}^{(d)}
\end{array}\right] \text { with } d=0,1,2 .
$$

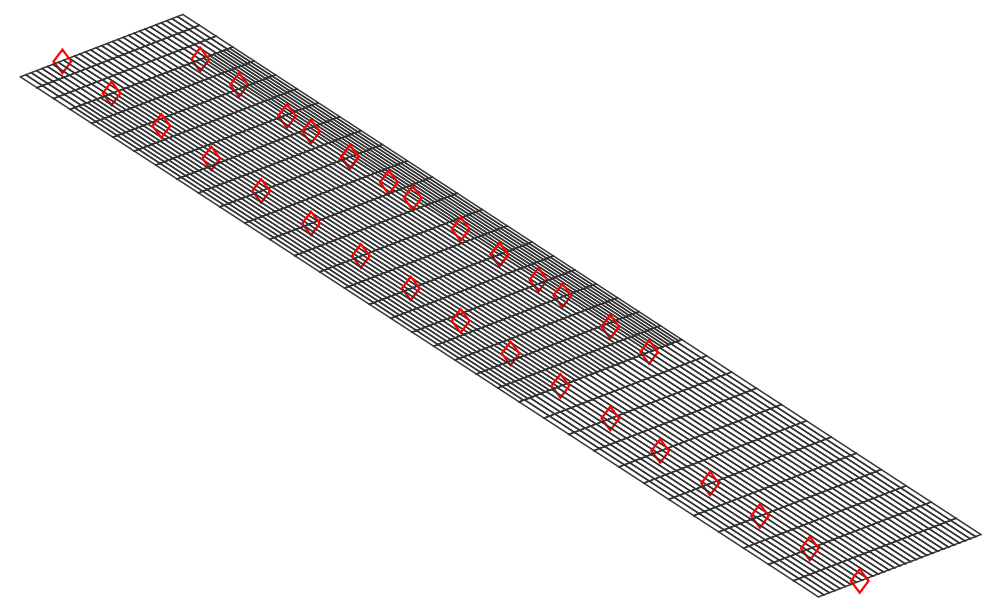

Fig. 2 Aerodynamic panel model with nodes of condensed FEM.

\section{Actuation}

\section{Trailing Edge Flaps}

In order to allow for an active GLA, the experimental flexible wing is augmented by three trailing edge flaps of equal size, each one driven by a $M K S H B L 990$ servo. The transfer function from the commanded to the actual flap deflection angle is identified by means of the actuator testbed depicted in Figure 3. For identification, sine sweeps of different amplitudes are commanded and the resulting flap angles are measured yielding the frequency response plotted 
in Figure 4. Clearly, a certain bandwidth limitation as well as time delay are visible. To approximate the measured frequency response for a maximum flap deflection of $\pm 10^{\circ}$, the coefficients $a_{j}, j=1, . ., 4$ of the transfer function

$$
G_{\text {approx }}(s)=\frac{a_{1} s+a_{2}}{s^{2}+a_{3} s+a_{4}}
$$

are determined by means of a least squares fit. As a result, the actual flap deflections are

$$
\delta=\left[\begin{array}{l}
\delta_{1} \\
\delta_{2} \\
\delta_{3}
\end{array}\right]=\left[\begin{array}{ccc}
G_{\text {approx }}(s) & 0 & 0 \\
0 & G_{\text {approx }}(s) & 0 \\
0 & 0 & G_{\text {approx }}(s)
\end{array}\right]\left[\begin{array}{l}
\delta_{1, \mathrm{cmd}} \\
\delta_{2, \mathrm{cmd}} \\
\delta_{3, \mathrm{cmd}}
\end{array}\right]=G_{\text {flap }}(s) \delta_{\mathrm{cmd}},
$$

where $\delta_{\mathrm{cmd}}$ summarizes the deflection commands for all flaps. Furthermore, a free play of $0.5^{\circ}$, a time delay of $10 \mathrm{~ms}$ and a maximum deflection rate of $1280 \% \mathrm{~s}$ are determined experimentally, which are not captured by the linear model derived here.

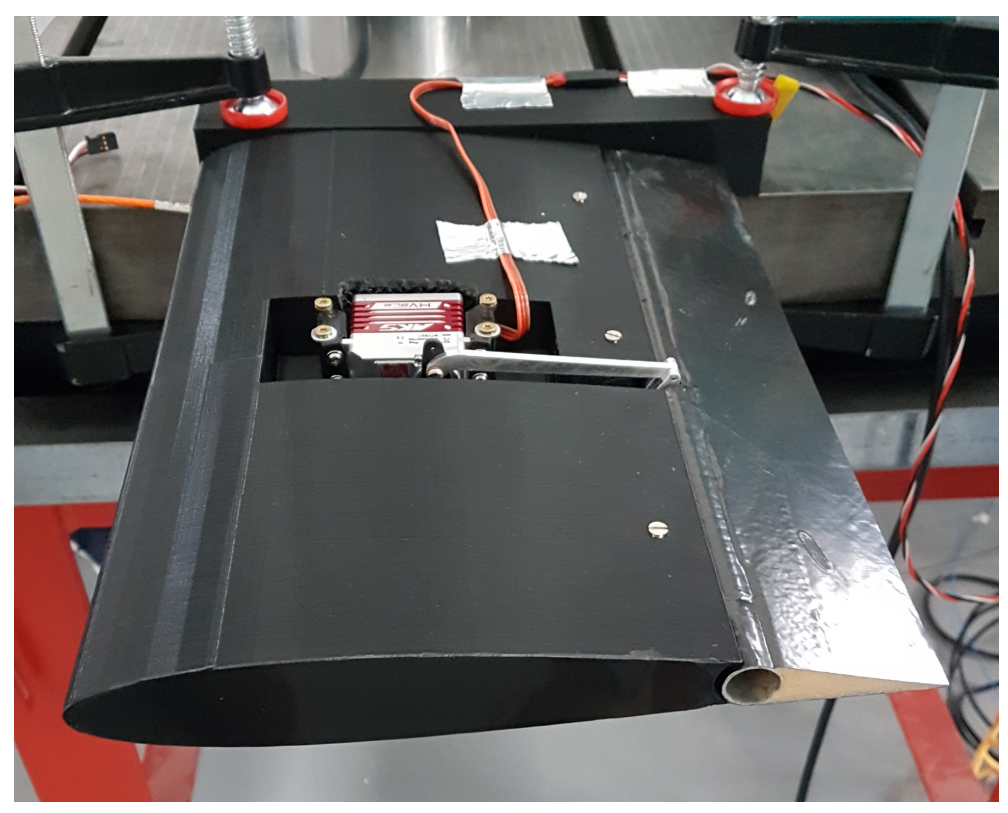

Fig. 3 Actuator identification testbed.

\section{Gust Simulation by Forced Pitching Motion}

In order to excite the experimental flexible wing, vertical gusts are simulated by a forced pitching motion around the quarter chord line. In the frequency range of interest, this kind of excitation is comparable to an external gust generator due to the high torsional stiffness of the wing. Pitching the wing adds an additional angle of attack, similarly to vertical gusts during flight. The pitching motion is executed by a Parker Torque Motor 190ST6M with a Compax3 I31T11 control unit. In order to precisely follow the commanded gust angle of attack $\alpha_{\mathrm{cmd}}^{\text {(gust) }}$, an appropriate pitching moment $p_{\text {pitch }}$ needs to be generated which is induced to the wing at its root. Transforming the induced pitching moment to modal coordinates by means of the transition matrix $\Phi_{\text {pitch }, h}$ yields

$$
p_{h}^{\mathrm{pitch}}=\Phi_{\mathrm{pitch}, h}^{T} G_{\mathrm{pitch}}(s) \alpha_{\mathrm{cmd}}^{\text {(gust) }},
$$

where $G_{\text {pitch }}(s)$ is a second order low pass filter describing the closed-loop behavior of the pitch excitation system. 

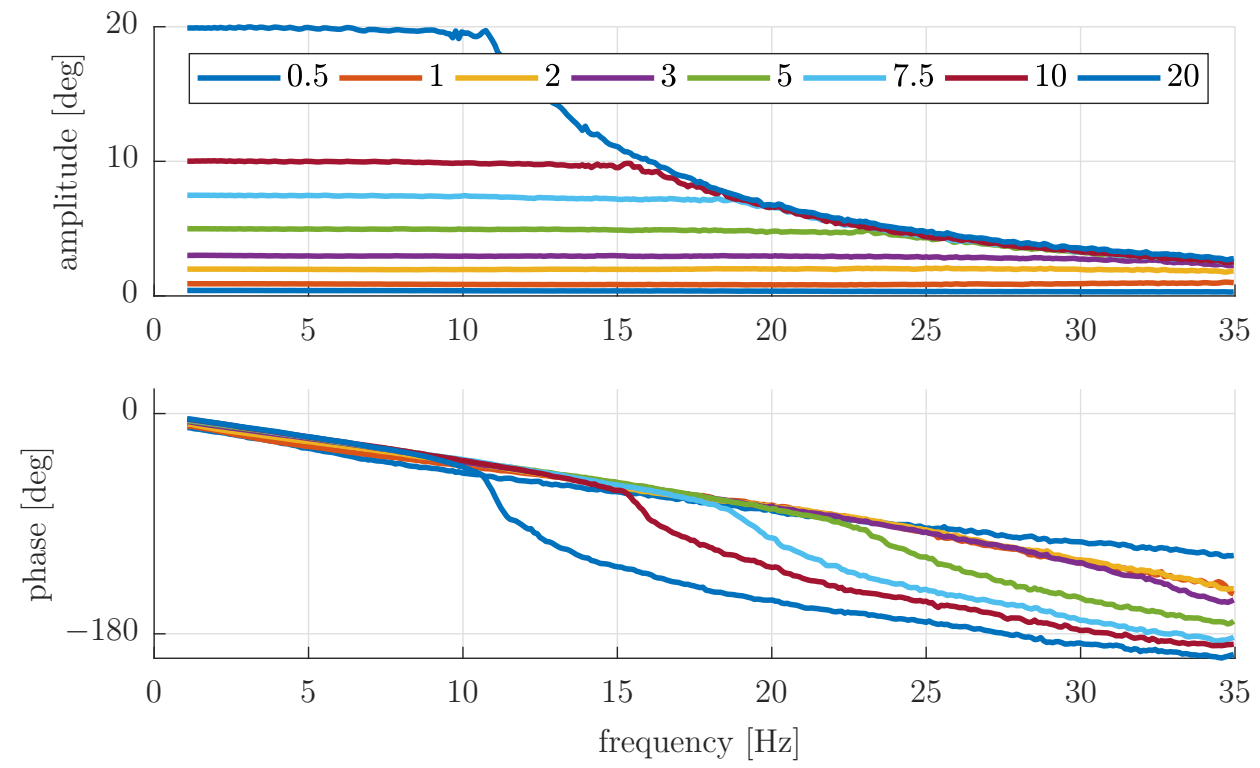

Fig. 4 Frequency response from commanded flap deflection to measured flap deflection for different amplitudes.

\section{Measurements}

\section{Acceleration Measurements}

For active GLA, eight vertical acceleration sensors are installed in the experimental flexible wing. The sensor positions are close to the flap positions and depicted in Figure 5. The acceleration measurements are computed as

$$
y_{\mathrm{acc}}=\Phi_{\mathrm{acc}, h} s^{2} x_{h}
$$

where the modal accelerations $\ddot{x}_{h}=s^{2} x_{h}$ are transformed to the corresponding sensor coordinate systems by means of the transition matrix $\Phi_{\text {acc, } h}$.

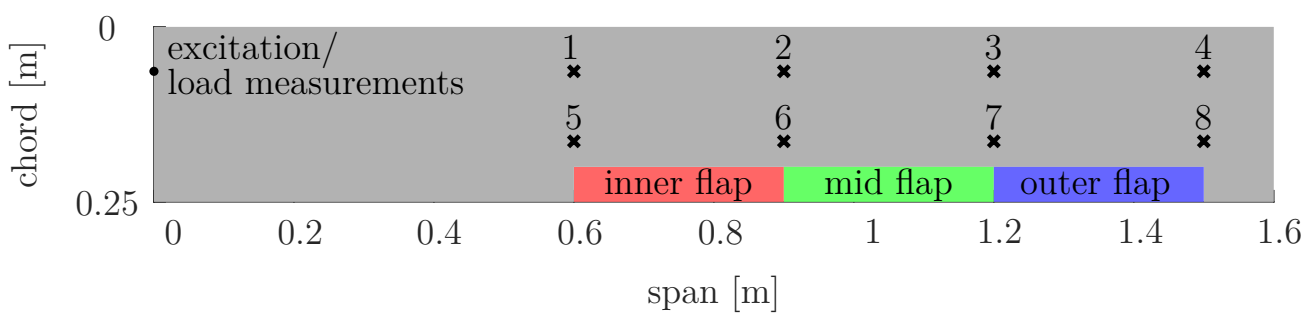

Fig. 5 Positions of flaps and sensors on the wing.

\section{Structural Loads Measurements}

The performance of the implemented GLA control system is evaluated in terms of the achieved wing root bending moment (WRBM) reduction. To measure the actual WRBM, a piezo balance is inserted between the wing root and the shaft of the pitch excitation system. By means of the force summation method [17], the occurring structural loads are given as

$$
p_{c}=p_{c}^{(\text {aero })}-p_{c}^{(\text {intert })}
$$

where $p_{c}^{\text {(intert) }}=T_{c, h} s^{2} x_{h}$ denote the inertial loads. 


\section{E. Model Integration}

In order to obtain the integrated aeroelastic model, the structural model from Section II-A is coupled with the aerodynamic model from Section II-B. In generalized coordinates, this is achieved by setting

$$
p_{h}^{(\mathrm{ext})}=p_{h}^{(\mathrm{aero})}+p_{h}^{(\mathrm{pitch})}
$$

where the external loads acting on the wing structure $p_{h}^{(\text {ext })}$ are actually a superposition of the aerodynamic loads $p_{h}^{\text {(aero) }}$ and the loads from pitch excitation $p_{h}^{\text {(pitch) }}$. Furthermore, the actuator dynamics described in Section II-C and the measurements described in Section II-D are added to the model. The interconnection of the individual models is depicted in Figure 6, where the resulting integrated model has 90 states and is linear time-invariant (LTI) for constant airspeeds.

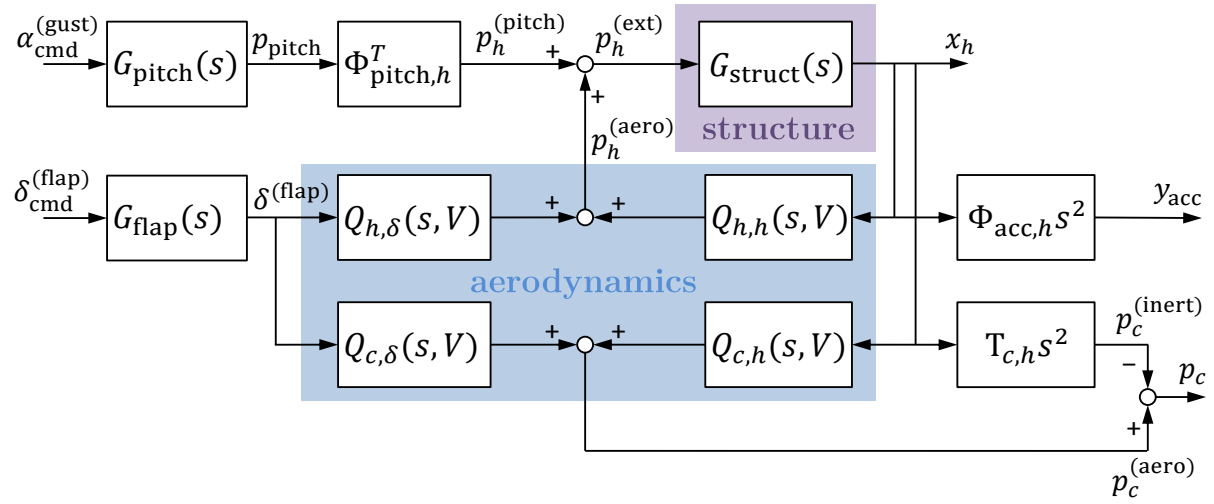

Fig. 6 Aeroelastic model including actuator dynamics and measurement outputs.

\section{F. Model Update}

Basically, the derived integrated aeroelastic model is required for the model-based control design procedure described in Section III. Since the performance of the controller greatly depends on the accuracy of the underlying simulation model, it is proposed to update individual parts as described in the following subsections. Based on the updated model, the controller is re-tuned to guarantee closed-loop stability and maintain a maximum controller performance.

\section{Mass model}

Before assembling the experimental wing, its components are weighted and added as point masses to the original FEM. The individual component masses are summarized in Table 1, where the total mass of the wing is approximately $3.6 \mathrm{~kg}$. Subsequently, a modal analysis is carried out again on the updated FEM yielding a new structural model.

\section{Table 1 Masses of individual components.}

\begin{tabular}{lr}
\hline \hline & mass (g) \\
\hline sensors (incl. cables) & 167 \\
actuators (incl. cables) & 260 \\
flaps (incl. mounting) & 300 \\
wing (incl. mounting) & 2840 \\
\hline total wing mass & 3567 \\
\hline \hline
\end{tabular}

\section{Modal test}

After assembling the experimental wing, a modal test is carried out in order to identify the actual structural modes. To that end, additional acceleration sensors are mounted on the wing and pitch excitation system measuring the response 
of the wing when it is excited at different locations with a modal hammer. Subsequently, the natural frequency, relative damping and the shape of the structural modes are obtained by means of an input-output modal analysis, see [18] and [19] for more details. In Table 2, the identified first four structural eigenmodes are listed, where the first wing bending mode is subject to be controlled. In order to accomplish an adequate match of theoretical and experimental modal results, parameters with the largest uncertainty in the FEM are adapted:

1) Fiber angles in the load carrying wing skins are altered by 1 to 2 degrees, considering variations in the hand layup process.

2) Adaptation of a degradation factor accounting for a stiffness reduction due to fiber ondulation.

3) Adaptation of the foam core density, considering manufacturing variations.

Eventually, another update of the structural model is obtained by carrying out a modal analysis on the adapted FEM.

Table 2 Updated structural modes.

\begin{tabular}{lcc}
\hline \hline mode name & frequency $(\mathrm{Hz})$ & damping $(\%)$ \\
\hline $1^{\text {st }}$ bending & 7.96 & 0.4 \\
$1^{\text {st }}$ in-plane & 29.10 & 0.5 \\
$2^{\text {nd }}$ bending & 42.20 & 0.7 \\
$1^{\text {st }}$ torsion & 82.70 & 1.9 \\
\hline \hline
\end{tabular}

Actuator model

Besides updating the structural model by correcting local mass and stiffness, also the flap actuation system installed on the actual wing is re-identified and updated in the model. To that end, the procedure from Section II-C is applied to each individual flap, where sine sweeps are used to identify the transfer function from the commanded to the actual flap deflection. Furthermore, the maximum deflection rate and free play are determined for each flap and given in Table 3. In the wind tunnel, the same procedure is repeated at different airspeeds to consider also real aerodynamic loads. However, the identified parameters changed only marginally which means that the power of the chosen actuators is sufficient.

Table 3 Updated parameters of the flap actuation system.

\begin{tabular}{lcc}
\hline \hline mode name & free play $\left(^{\circ}\right)$ & max. rate $(\% / \mathrm{s})$ \\
\hline inner flap & 1.5 & 1054 \\
mid flap & 0.4 & 948 \\
outer flap & 1.2 & 805 \\
\hline \hline
\end{tabular}

\section{Aeroelastic Mode Control}

In Section II, an aeroelastic model of the experimental wing is derived which depends on the true airspeed $V$. To obtain the shape, frequency and damping of the individual aeroelastic modes, the airspeed is fixed at different values and a modal decomposition is carried out on the resulting LTI systems. As expected, the lightly damped first and second wing bending modes clearly dominate the WRBM, which can also be seen in the frequency response given in Figure 7. Since the second wing bending mode is marginally excited during gust encounter due to its high natural frequency, the objective for GLA controller design is to increase the damping of the first wing bending mode. To that end, the modal control approach from [11] is applied, where the first wing bending mode is first isolated by blending the control inputs and measurement outputs. Here, the deflection commands $\delta_{\mathrm{cmd}}^{\text {(flap }}$ of the $n_{u}=3$ trailing edge flaps are considered as control inputs and the $n_{y}=8$ vertical acceleration sensor signals $y_{\text {acc }}$ are considered as measurement outputs. Based on the system with blended inputs and outputs, a SISO controller design is enabled, where the goal is to increase the damping of the isolated mode. Since the shape of the first wing bending mode varies only slightly within the considered airspeed range, the corresponding input and output blending vectors $k_{u} \in \mathbb{R}^{n_{u}}$ and $k_{y} \in \mathbb{R}^{n_{y}}$ are computed at a single airspeed and held constant for all airspeeds. In contrast to that, the gains of the SISO controller $c(s, V)$ are scheduled 
with $V$ to achieve a better controller performance. The resulting closed-loop interconnection is depicted in Figure 8 , where the dashed line encircles the gain-scheduled controller

$$
K(s, V)=k_{u} c(s, V) k_{y}^{T}
$$

Note that the transfer function matrices of the controller $K(s, V)$ and the plant $G(s, V)$ are defined only for fixed airspeeds $V$. The overall controller design procedure is schematically visualized in Figure 9, where the individual parts are described in the following subsections.

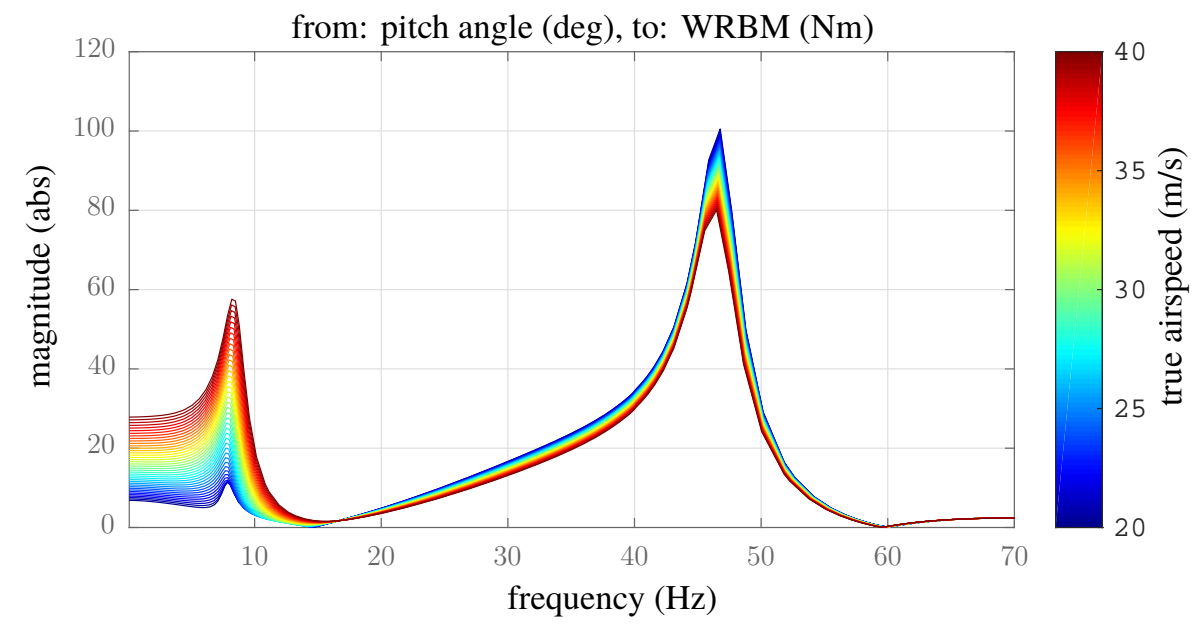

Fig. 7 Frequency response of updated model from pitch angle to WRBM at different airspeeds.

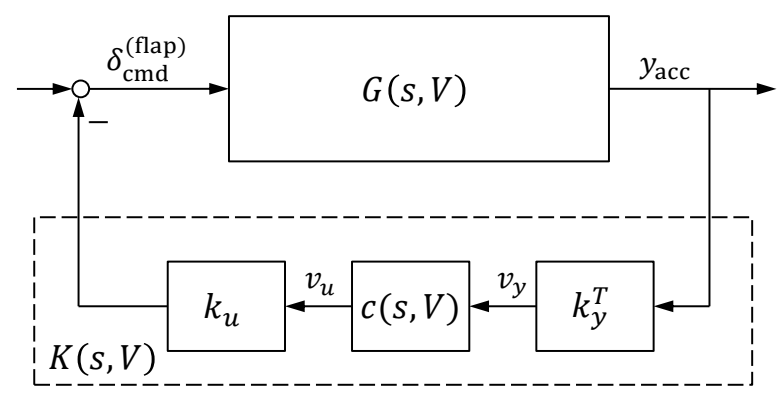

Fig. 8 Closed-loop interconnection of the plant $G(s, V)$ with the controller $K(s, V)$.

State Space
Model $\rightarrow \begin{gathered}\text { Modal } \\ \text { Decomposition }\end{gathered} \rightarrow \begin{gathered}\text { Input / Output } \\ \text { Blending }\end{gathered} \rightarrow$ S $\begin{aligned} & \text { SISO Controller } \\ & \text { Design / Tuning }\end{aligned} \rightarrow \begin{gathered}\text { Controller } \\ \text { Verification }\end{gathered}$

Fig. 9 Controller design procedure.

\section{A. Modal Decomposition}

For a fixed airspeed $V$, the described aeroelastic model yields an LTI system which can be decomposed as

$$
G(s, V)=\sum_{i=1}^{n_{i}} M_{i}(s, V)+D
$$


where the individual modes $i=1, \ldots, n_{i}$ are given as

$$
M_{i}(s, V)= \begin{cases}\frac{R_{i}}{s-p_{i}} & \text { if } \mathfrak{J}\left(p_{i}\right)=0 \\ \frac{R_{i}}{s-p_{i}}+\frac{\bar{R}_{i}}{s-\bar{p}_{i}} & \text { otherwise. }\end{cases}
$$

According to Equation (13), a mode $i$ is either described by a single real pole $p_{i}$ with an imaginary part $\mathfrak{J}\left(p_{i}\right)=0$ or a conjugate complex pole pair $p_{i}$ and $\bar{p}_{i}$. Each pole $p_{i}$ is associated with a residue $R_{i} \in \mathbb{C}^{n_{y} \times n_{u}}$, where for a real pole, the corresponding residue is real, and for a conjugate complex pole pair the residues are conjugate complex. Note that the given poles as well as residues also depend on the fixed true airspeed $V$, which is not explicitly given here for ease of notation.

\section{B. $\mathcal{H}_{2}$-optimal Blending of Inputs and Outputs for Modal Control}

The $\mathcal{H}_{2}$-norm of an individual aeroelastic mode is a well-suited metric to quantify its controllability and observability in a combined way [11]. Based on this measure, $\mathcal{H}_{2}$-optimal input and output blending vectors $k_{u}$ and $k_{y}$ for controlling the first wing bending mode $M(s, V)$ are derived by solving

$$
\begin{array}{ll}
\underset{k_{u} \in \mathbb{R}^{n_{u}}, k_{y} \in \mathbb{R}^{n_{y}}}{\operatorname{maximize}} & \left\|k_{y}^{T} M(s, V) k_{u}\right\|_{\mathcal{H}_{2}} \\
\text { subject to } & \left\|k_{u}\right\|_{2}=1 \\
& \left\|k_{y}\right\|_{2}=1 .
\end{array}
$$

The blending vectors $k_{u}$ and $k_{y}$, which are required to be real in order to be realizable, can be seen as input and output directions of unity length enforced by the constraints (14b) and (14c). The constrained optimization problem (14a) - (14c) has $n_{u}+n_{y}$ optimization variables and can be solved by reformulating it as an unconstrained optimization problem in a single variable, see [11] for more details. Since the $\mathcal{H}_{2}$-optimal blending vectors change only marginally for different airspeeds, the ones computed for $V=40 \mathrm{~m} / \mathrm{s}$ are used at all airspeeds.

Blending the inputs and outputs of the aeroelastic model from Section II-E results in a SISO system, for which the frequency response is depicted in Figure 10. The first wing bending mode with a natural frequency around $8 \mathrm{~Hz}$ is clearly emphasized while the contributions of higher frequent modes are minor. Hence, the desired isolation of the first wing bending mode is achieved, which enables a SISO controller design described in the following subsection.

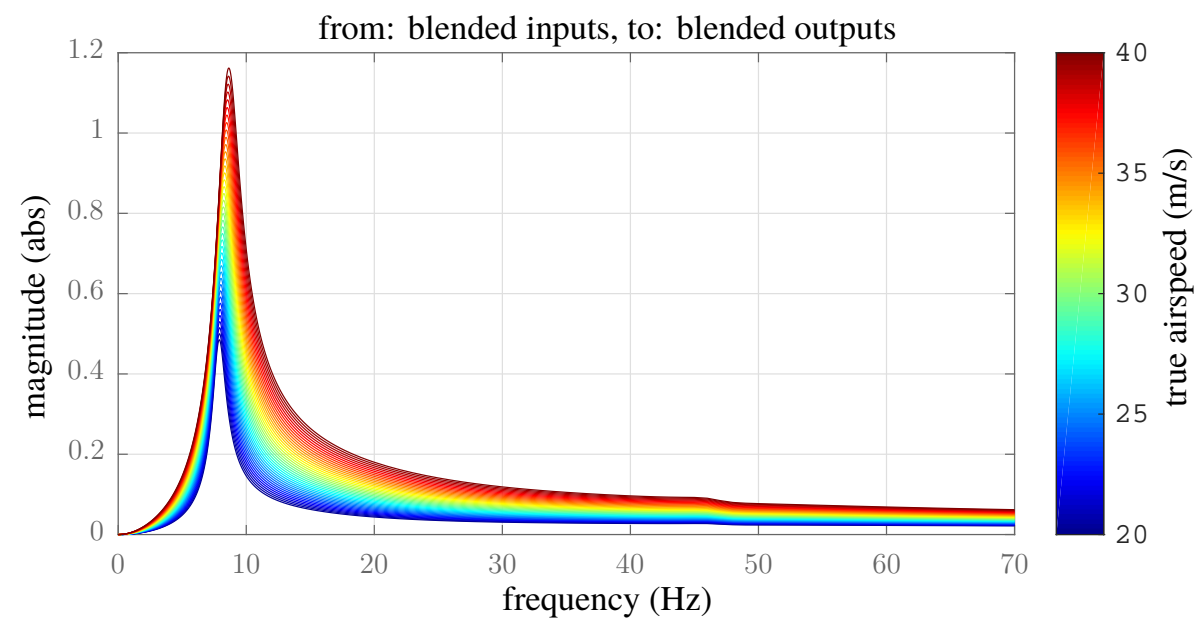

Fig. 10 Comparison of frequency response from blended inputs to blended outputs (open-loop).

\section{SISO Controller Design and Tuning}

After blending control inputs and measurement outputs of the experimental flexible wing, a SISO controller is designed to damp the first wing bending mode and thereby reduce the WRBM. The SISO controller chosen here is 
given as

$$
c(s, V)=k_{p}(V)+k_{i}(V) \frac{1}{s},
$$

where the proportional and integral gains $k_{p}(V): \mathbb{R} \rightarrow \mathbb{R}$ and $k_{i}(V): \mathbb{R} \rightarrow \mathbb{R}$ are scheduled with true airspeed $V$. The controller gains are tuned manually at fixed true airspeeds of $20 \mathrm{~m} / \mathrm{s}, 30 \mathrm{~m} / \mathrm{s}$ and $40 \mathrm{~m} / \mathrm{s}$ and linearely interpolated inbetween. With the goal to maximize the damping of the first wing bending mode, the SISO controller is tuned such that actuator saturation does not occur for the considered gust excitations. Additionally, classical gain and phase margins of at least $6 \mathrm{~dB}$ and $45 \mathrm{deg}$ are taken into account, respecitvely.

The achieved damping of the first wing bending mode is depicted in Figure 11. It can be seen that the relative damping is increased by a factor of more than five at higher airspeeds while it is not even tripeled at lower airspeeds. The reason for this is the efficiency of the flaps, which also increases with airspeed. Furthermore, the poles of the remaining aeroelastic modes are hardly affected when closing the loop, which indicates a good isolation of the target mode applying the input and output blends. Besides the increase in modal damping, the performance of the designed GLA system is evaluated in terms of the achieved WRBM reduction during vertical gust encounter. To that end, different types of gusts are simulated by the pitch excitation system, where the obtained results are given in the next section.

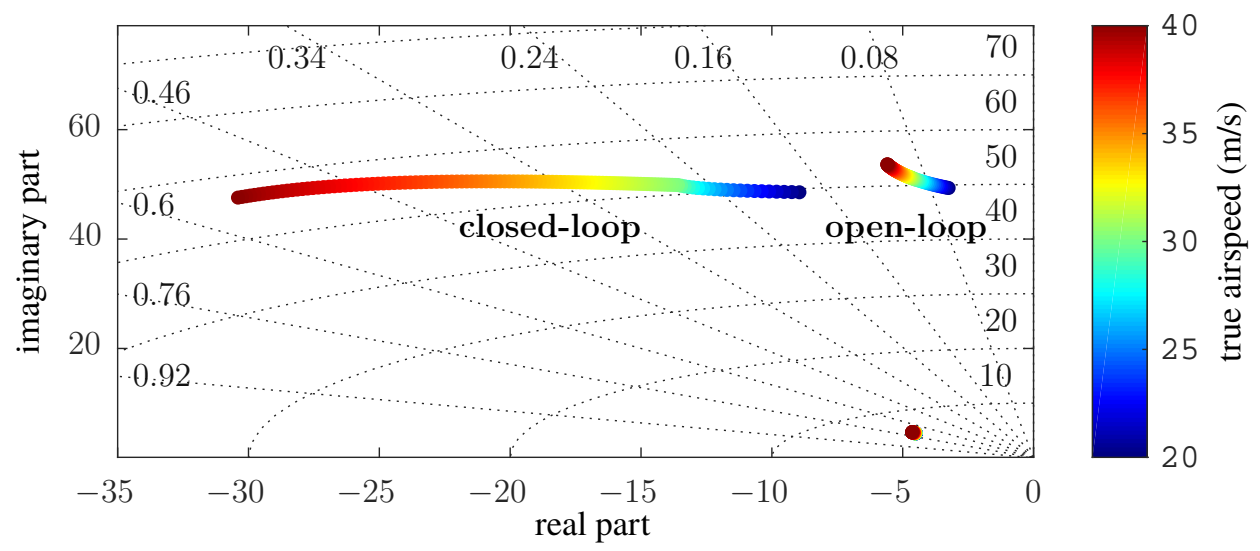

Fig. 11 Poles of the open-loop and closed-loop aeroelastic model for different airspeeds.

\section{Experimental Results}

The GLA controller derived in Section III is verified at the Crosswind Simulation Facility (Seitenwindkanal Göttingen, SWG) of the German Aerospace Center (DLR) in Göttingen. The maximum flow velocity of the closed-circuit wind tunnel is $65 \mathrm{~m} / \mathrm{s}$ and the dimension of the test section is $2.4 \mathrm{~m}$ (width) by $1.6 \mathrm{~m}$ (height). The flexible wing, depicted in Figure 12, is mounted on the pitch excitation system which is outside of the wind tunnel. After validating the derived aeroelastic model, the loop is closed and the acutal GLA controller performance is determined for different gust excitations.

\section{A. Model Validation}

For aeroelastic model validation, sweep excitations are performed at 20,30 and $40 \mathrm{~m} / \mathrm{s}$ true airspeed yielding the transfer functions from pitch angle and individual flap deflection angles to vertical accelerations and loads measurements. The experimental results correspond well to the simulation results as it can be seen in Figure 13. The largest mismatch is visible in the channel from the blended inputs (flap deflections) to the blended outputs (acceleration measurements), where it is assumed that free play in the pitch excitation system yields smaller amplitudes than expected. During the experiments, the aeroelastic modes of interest are directly identified by means of a real-time capable output-only modal analysis, see [20] and [21] for more details. For the first wing bending mode, the natural frequency remains almost constant while its damping increases with airspeed. This also corresponds well to the open-loop simulation results as compared in Table 4. 


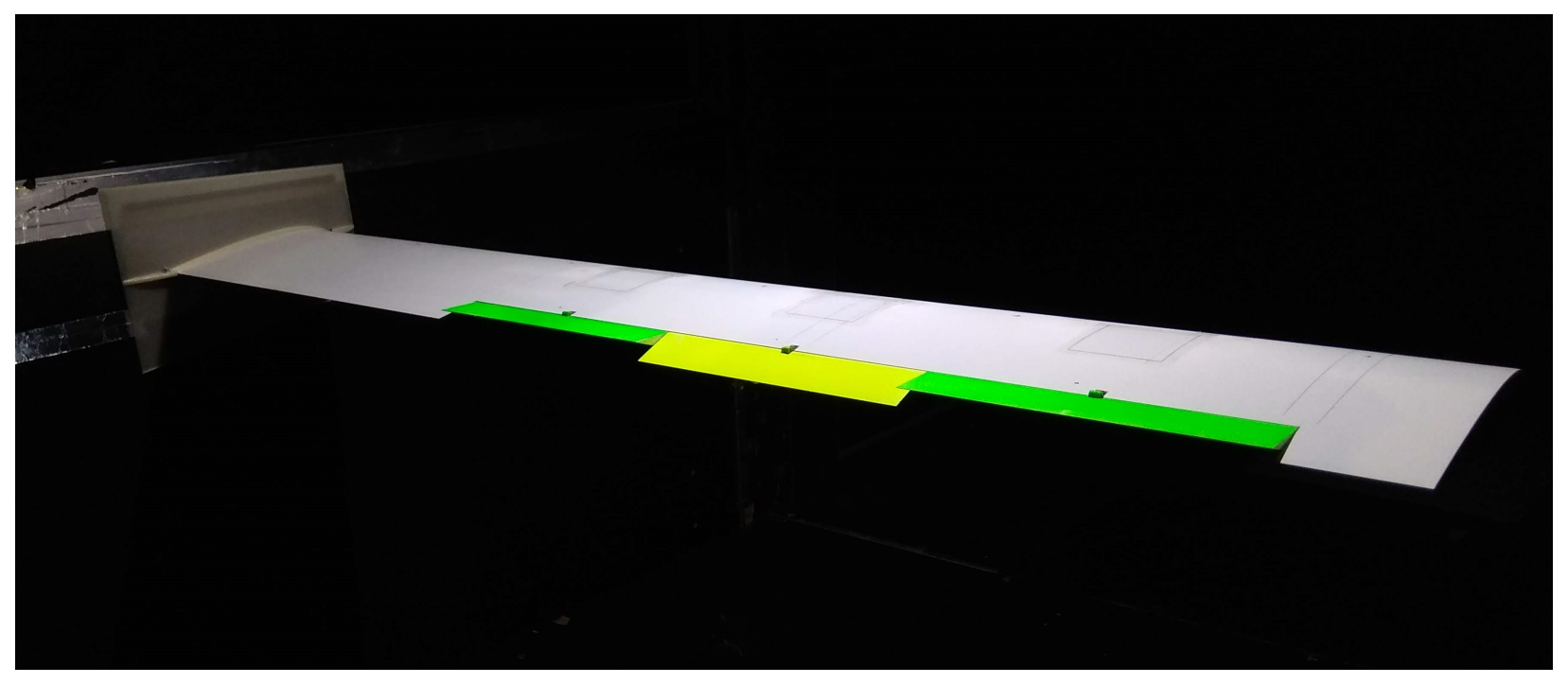

Fig. 12 Experimental wing with three trailing edge flaps in the windtunnel.

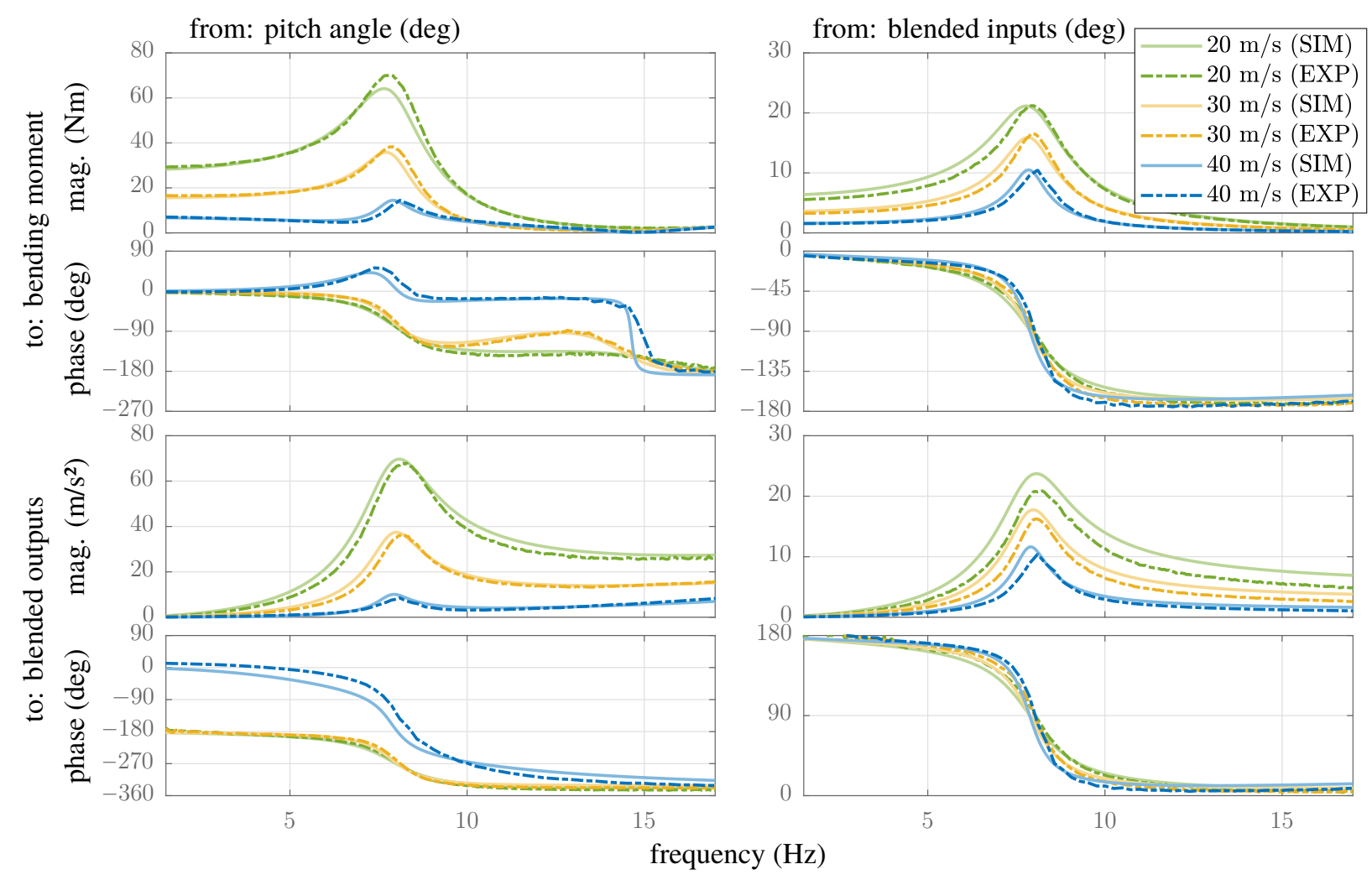

Fig. 13 Comparison of aeroelastic simulation model (SIM) and experimentally identified model (EXP). 
Table 4 Comparison of natural frequency and relative damping of the first bending mode in simulation (SIM) and experiment (EXP) for the open-loop and closed-loop case.

\begin{tabular}{|c|c|c|c|c|}
\hline \multirow[t]{3}{*}{ true airspeed $(\mathrm{m} / \mathrm{s})$} & \multicolumn{2}{|c|}{ natural frequency $(\mathrm{Hz})$} & \multicolumn{2}{|c|}{ relative damping (\%) } \\
\hline & open-loop & closed-loop & open-loop & closed-loop \\
\hline & SIM / EXP & SIM / EXP & SIM / EXP & SIM / EXP \\
\hline 20 & $7.9 / 8.1$ & $7.9 / 8.2$ & $6.6 / 4.9$ & $18.1 / 11.0$ \\
\hline 30 & $8.2 / 8.0$ & $8.2 / 7.8$ & $8.9 / 8.4$ & $26.2 / 21.1$ \\
\hline 40 & $8.6 / 8.0$ & $9.0 / 8.1$ & $10.4 / 11.0$ & $53.8 / 34.4$ \\
\hline
\end{tabular}

\section{B. Controller Verification}

In order to verify the GLA capability of the controller derived in Section III, the flexible wing is excited by stochastic and discrete gusts. In the stochastic case, a band-limited noise between $1-30 \mathrm{~Hz}$ is commanded to the pitch excitation system. The intensity of the noise is chosen such that a maximum WRBM of $120 \mathrm{~N} \mathrm{~m}$ is not exceeded and hence varies with airspeed. The root mean square (RMS) of the WRBM is reduced by $28 \%$ at $V=40 \mathrm{~m} / \mathrm{s}$ while at $V=20 \mathrm{~m} / \mathrm{s}$, a reduction of only $10 \%$ is achieved since flap efficiency is decreased at low airspeeds.

As discrete gust excitations, "1-cos" gusts according to the certification requirements [22] and [23] are applied. Different intensities and gust gradient distances are tested, where the WRBM becomes a maximum when the gust frequency coincides with the natural frequency of the first wing bending mode. Plotting the WRBM for this special case in Figure 15, it can be seen that both the peak and the settling time are reduced considerably when the controller is turned on (closed-loop). The corresponding flap deflection commands are depicted in Figure 16, where it can be seen that the outer flap is deflected most. In comparison to that, the inner flap deflection commands are within free play meaning that in reality, the inner flap is not deflected. Obviously, this yields a loss in GLA performance which is confirmed in nonlinear simulations.

Furthermore, sweep excitations are used to identify the closed-loop transfer function from the pitch angle to the WRBM. In Figure 14, the reduction of the WRBM is clearly visible and results from damping the first wing bending mode. The actual increase in modal damping is again identified online (and verified offline), where the corresponding values are given in Table 4. Compared to the simulation results, however, the increased damping of the first wing bending mode is considerably smaller than expected for all considered airspeeds. The main reason for this is again the large free play of the flap actuation system as given in Table 3. All in all, the designed controller provides a good GLA performance which may be further improved by reducing free play.

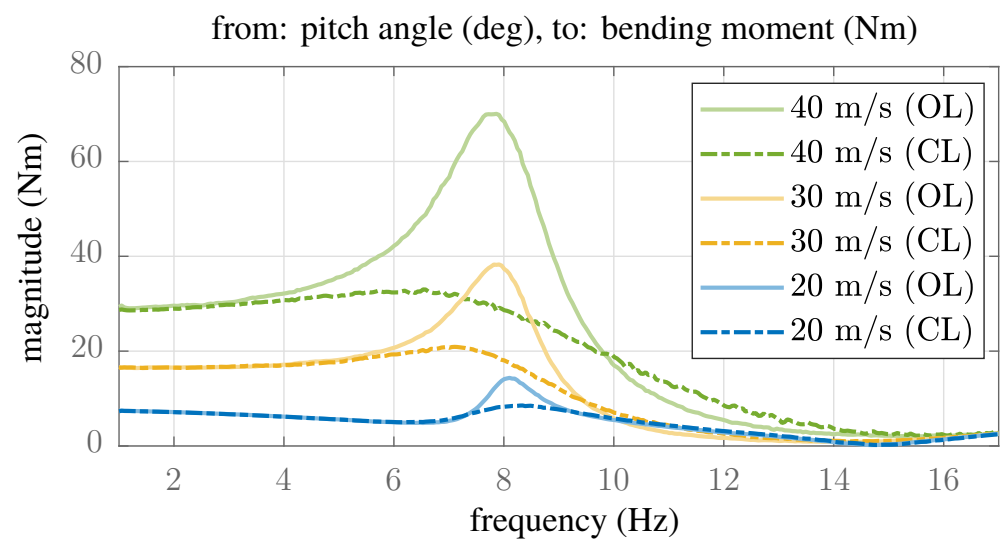

Fig. 14 Comparison of identified open-loop (OL) and closed-loop (CL) frequency response at different airspeeds. 


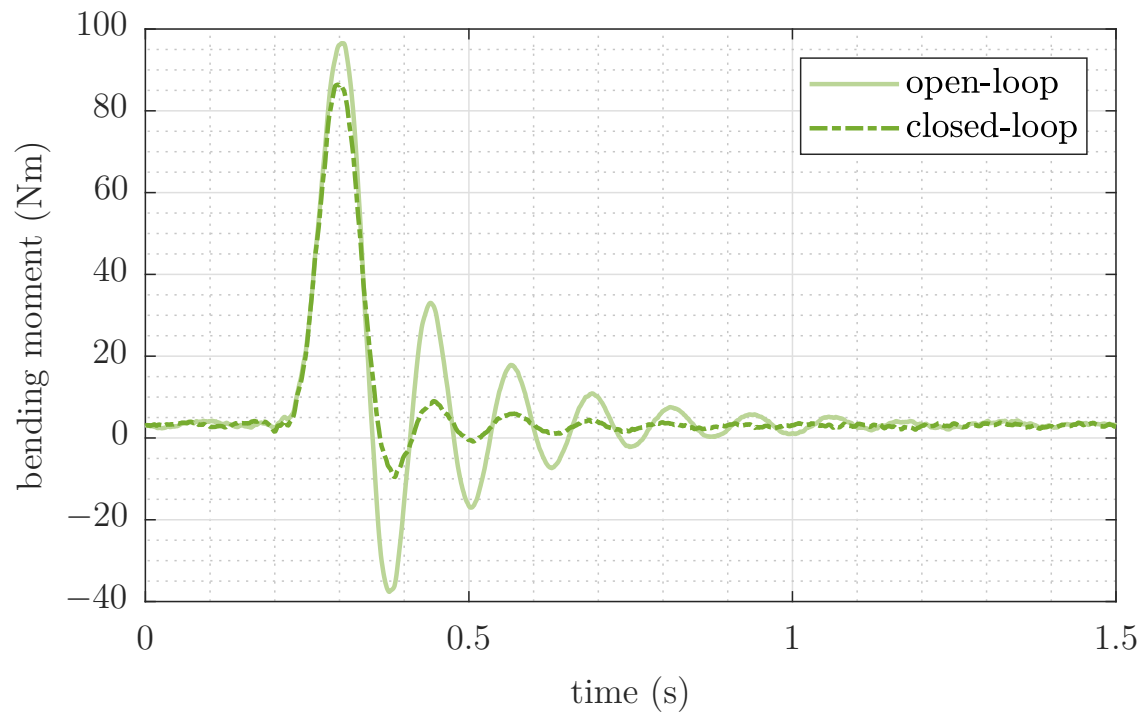

Fig. 15 Comparison of WRBM for "1-cos" gust excitation at $V=40 \mathrm{~m} / \mathrm{s}$ with and without GLA.

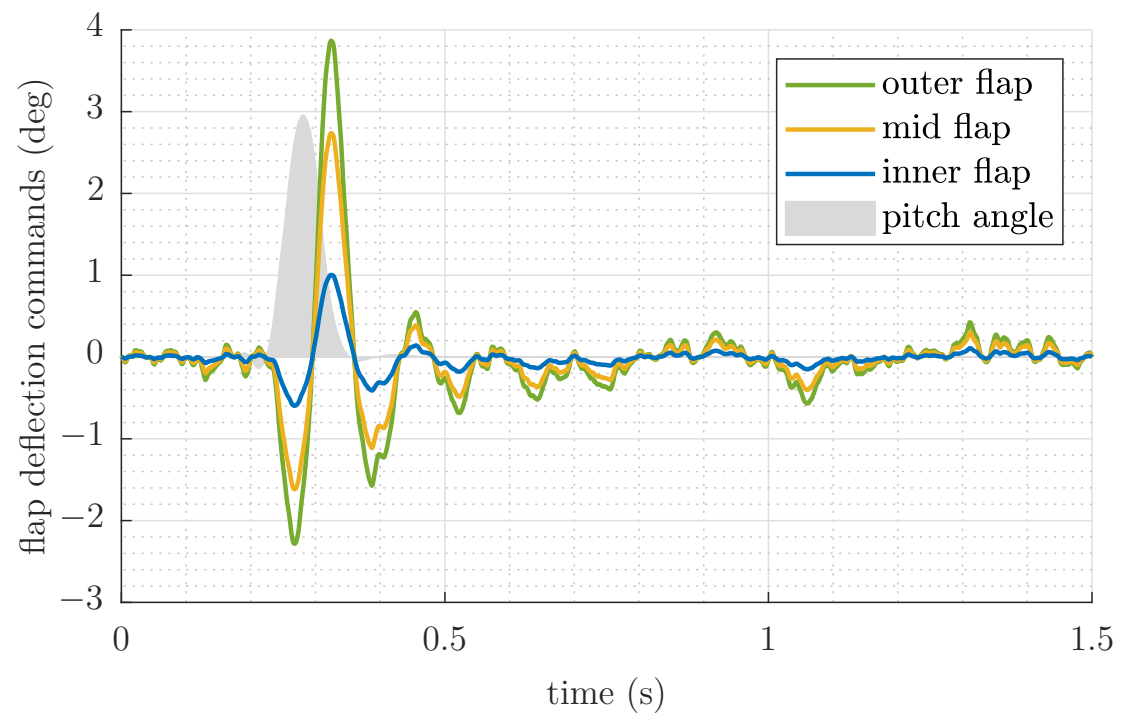

Fig. 16 Flap deflection commands for "1-cos" gust excitation at $\mathrm{V}=\mathbf{4 0} \mathrm{m} / \mathrm{s}$. 


\section{Conclusions and Outlook}

In this paper, an advanced GLA control system is developed and verified in a wind tunnel test campaign. To that end, multiple acceleration measurements are fed back to three trailing edge flaps, where an $\mathcal{H}_{2}$-optimal blending approach is applied to isolate the first wing bending mode. Based on that, the isolated mode can be damped efficiently by means of a SISO controller which is scheduled with airspeed. For the model-based control design approach, an integrated aeroelastic model of the experimental flexible wing is derived which is validated during the wind tunnel test campaign. The actual GLA controller performance is evaluated for different gust excitations and confirms the great potential of the proposed control approach.

\section{Acknowledgments}

The authors thank (in alphabetical order) Heiko Bölken, Maria Fröhlich, Ramesh Konatala, Yasser Meddaikar, Tobias Meier, Keith Soal, Christian Stieg and Bernd Will for their great support making the wind tunnel experiment a success. This work was founded in the frame of the German Aeronautical Research Program project KonteKst.

\section{References}

[1] Transport, Energy and $\mathrm{CO}_{2}$, International Energy Agency, 2009.

[2] Regan, C. D., and Jutte, C. V., "Survey of Applications of Active Control Technology for Gust Alleviation and New Challenges for Lighter-weight Aircraft," Tech. Rep. NASA/TM-2012-216008, NASA, 2012.

[3] von Kármán, T., and Edson, L., The wind and beyond, Little, Brown \& Company, 1967.

[4] Hansen, J., Aeronautics, U. S. N., and Office, S. A. H., The Wind and Beyond: A Documentary Journey Into the History of Aerodynamics in America, No. v. 1 in NASA SP, National Aeronautics and Space Administration, NASA History Office, Office of External Relations, 2003.

[5] Wildschek, A., "An adaptive feed-forward controller for active wing bending vibration alleviation on large transport aircraft," Ph.D. thesis, Technische Universität München, 2009.

[6] Vartio, E., Shimko, A., Tilmann, C., and Flick, P., "Structural modal control and gust load alleviation for a sensorcraft concept," 46th AIAA/ASME/ASCE/AHS/ASC Structures, Structural Dynamics and Materials Conference, 2005, p. 1946.

[7] Mangalam, A., Mangalam, S., and Flick, P., "Unsteady Aerodynamic Observables for Gust Load Alleviation," 49th AIAA/ASME/ASCE/AHS/ASC Structures, Structural Dynamics, and Materials Conference, 16th AIAA/ASME/AHS Adaptive Structures Conference, 10th AIAA Non-Deterministic Approaches Conference, 9th AIAA Gossamer Spacecraft Forum, 4th AIAA Multidisciplinary Design Optimization Specialists Conference, 2008, p. 1725.

[8] Scott, R., Coulson, D., Castelluccio, M., and Heeg, J., "Aeroservoelastic Wind-Tunnel Tests of a Free-Flying, Joined-Wing SensorCraft Model for Gust Load Alleviation," 52nd AIAA/ASME/ASCE/AHS/ASC Structures, Structural Dynamics and Materials Conference 19th AIAA/ASME/AHS Adaptive Structures Conference 13t, 2011, p. 1960.

[9] Reckzeh, D., "Multifunctional wing moveables design of the A350XWB and the way to future concepts," 29th Congress of the International Council of the Aeronautical Sciences (ICAS), St. Petersburg, 2014, pp. 1-10.

[10] Varga, A., "Computational challenges in flight control design," IEEE International Symposium on computer Aided control System Design, Hawaii, USA, 1999, pp. 1-6.

[11] Pusch, M., "Aeroelastic Mode Control using $\mathcal{H}_{2}$-optimal Blends for Inputs and Outputs," AIAA Guidance, Navigation, and Control Conference, Orlando, FL, USA, 2018, pp. 1-13.

[12] Kier, T., and Looye, G., "Unifying manoeuvre and gust loads analysis models," International Forum on Aeroelastisity and Dynamics. Seattle, USA, 2009, pp. 1-14.

[13] Dillinger, J., Klimmek, T., Abdalla, M., and Gürdal, Z., "Stiffness Optimization of Composite Wings with Aeroelastic Constraints," Journal of Aircraft, Vol. 50, No. 4, 2013, pp. 1159-1168.

[14] Meddaikar, M. Y., Dillinger, J., Ritter, M. R., and Govers, Y., “Optimization \& Testing of Aeroelastically-Tailored Forward Swept Wings," IFASD 2017 - International Forum on Aeroelasticity and Structural Dynamics, 2017, pp. 1-13.

[15] Rodden, W., and Johnson, E., MSC.Nastran Version 68, Aeroelastic Analysis and User's Guide, 2004. 
[16] Roger, K. L., “Airplane Math Modelling Methods for Active Control Design,” Structures and Materials Panel, 1977.

[17] Bisplinghoff, R., Ashley, H., and Halfman, R., "Aeroelasticity,” 1955.

[18] Govers, Y., Böswald, M., Lubrina, P., Giclais, S., Stephan, C., and Botargues, N., "Airbus A350XWB ground vibration testing: Efficient techniques for customer oriented on-site modal identification," Proceedings of the International Conference on Noise and Vibration Engineering. KU Leuven, Belgium, 2014, pp. 2503-2516.

[19] Böswald, M., Schwochow, J., Jelicic, G., and Govers, Y., "New Concepts for Ground and Flight Vibration Testing of Aircraft based on Output-Only Modal Analysis," IOMAC 2017 - 7th International Operational Modal Analysis Conference, Shaker Verlag, Ingolstadt, Germany, 2017, pp. 15-34.

[20] Jelicic, G., Schwochow, J., Govers, Y., Hebler, A., and Böswald, M., "Real-time assessment of flutter stability based on automated output-only modal analysis," Proceedings of the International Conference on Noise and Vibration Engineering. KU Leuven, Belgium, 2014, pp. 3693-3706.

[21] Jelicic, G., Schwochow, J., Govers, Y., Sinske, J., Buchbach, R., and Springer, J., "Online monitoring of aircraft modal parameters during flight test based on permanent output-only modal analysis," 58th AIAA/ASCE/AHS/ASC Structures, Structural Dynamics, and Materials Conference, 2017, p. 1825.

[22] Federal Aviation Regulations Part 25, Airworthiness Standards: Transport Category, Federal Aviation Administration, 2015.

[23] Certification Specifications and Acceptable Means of Compliance for Large Aeroplanes, CS-25, Amendment 16, European Aviation Safety Agency, 2015. 